Research paper

\title{
Age-related differences in EEG beta activity during an assessment of ankle proprioception
}

\author{
Diana R. Toledo ${ }^{\mathrm{a}, *}$, José A. Barela ${ }^{\mathrm{b}, \mathrm{c}}$, Gilberto M. Manzano ${ }^{\mathrm{d}}$, André F. Kohn ${ }^{\mathrm{a}}$ \\ a Neuroscience Program and Biomedical Engineering Laboratory, University of São Paulo, EPUSP, PTC, São Paulo, Brazil \\ ${ }^{\mathrm{b}}$ Institute of Physical Activity and Sport Sciences, Cruzeiro do Sul University, São Paulo, Brazil \\ c Institute of Biosciences, São Paulo State University, Rio Claro, Brazil \\ ${ }^{\mathrm{d}}$ Division of Neurology, Federal University of São Paulo, São Paulo, Brazil
}

\section{H I G H L I G H T S}

- RT to kinesthetic perception was temporally related to the beta activity for both YA and OA groups.

- Delayed RT and ERD in OA may be related to impairment in afferent pathways.

- Active inhibition of the cortex (ERS) after motor response was observed only for OA.

\section{A R T I C L E I N F O}

\section{Article history:}

Received 5 March 2016

Received in revised form 4 April 2016

Accepted 12 April 2016

Available online 13 April 2016

\section{Keywords:}

Event-related (de-)synchronization (ERD/S)

Slow passive movement

Response time

Proprioception

Aging

Electroencephalography

\begin{abstract}
A B S T R A C T
The aim of this work was to compare cortical beta oscillatory activity between young (YA) and older (OA) adults during the assessment of ankle proprioception. We analyzed the response time (RT) to kinesthetic perception and beta event-related desynchronization/synchronization (ERD/ERS) in response to passive ankle movement applied at a slow speed, $0.5^{\circ} / \mathrm{s}$. The relationship between ERD/ERS and RT was investigated by classifying the signals into fast-, medium-, and slow-RT. The results showed a temporal relationship between beta oscillation changes and RT for both groups, i.e., earlier ERD and ERS were obtained for trials with faster response time. ERD was larger and delayed in OA compared to the YA, and beta ERS was present only for OA. These findings suggest that a less efficient proprioceptive signaling reaching the brain of OA requires a higher level of brain processing and hence the differences in ERD potentials between YA and OA. Furthermore, the occurrence of ERS in OA might represent a compensatory strategy of active cortical resetting for adequate sensorimotor behavior due to the age-related reduced peripheral input and neuromuscular impairments. Altered balance between excitatory and inhibitory intracortical activity in older adults presumably explains the changes in beta oscillations.
\end{abstract}

(c) 2016 Elsevier Ireland Ltd. All rights reserved.
Age-related deterioration in the musculoskeletal and neuronal systems, in addition to reduced levels of selective attention and working memory, can lead to a decline of sensorimotor performance in older adults. Consequently, neuronal compensatory mechanisms such as activation of additional cortical areas [1-3] and increased cortical beta activity [4] are suggested to occur in older adults in an attempt to effectively perform specific tasks. Extra-activation could be understood as a functional reorganization and/or a redistribution of functional networks.

\footnotetext{
* Corresponding author at: Biomedical Engineering Laboratory, University of São Paulo EPUSP, PTC, Avenida Professor Luciano Gualberto, Travessa 3, n. 158, São Paulo, SP 05508-900, Brazil.

E-mail address: dianatoledo12@hotmail.com (D.R. Toledo).
}

Alternatively, reduced deactivation of cortical areas during motor tasks might indicate impaired modulatory capacity, wherein changes in inhibitory intracortical neural circuits could result in loss of performance $[5,6]$. The interpretation of specific changes in cortical oscillatory activity recorded at the scalp is not straightforward since the pattern of neuronal activity can be influenced by several factors. Some of these factors include the cognitive demand of the specific task, the movement characteristics, the age of the subjects, the integrity of the neuromuscular structures and the levels of excitation and inhibition between several interconnected cortical areas.

The balance between excitatory and inhibitory cortical activity helps to define and to constrain the possible neural processing and neural adaptations that may occur in the nervous system $[7,8]$. For this reason, it is relevant to study to what degree unbalance 
Table 1

Mean (M) and standard deviation (SD) values of age and anthropometric features for the young adult (YA) and older adult (OA) groups.

\begin{tabular}{rlrll}
\hline & & Age & Body Weight $(\mathrm{kg})$ & Stature $(\mathrm{m})$ \\
\hline YA & M & 28.53 & 68.16 & 1.67 \\
& SD & 3.03 & 11.97 & 0.08 \\
OA & M & 70.31 & 70.31 & 1.62 \\
& SD & 4.14 & 11.71 & 0.10 \\
\hline
\end{tabular}

of cortical activities occurs in the aged brain. Such information might provide meanings of how to prevent or revert functional sensorimotor deficits. A recent study from our laboratory combined electroencephalography (EEG) and response time (RT) task during brisk passive ankle movement and showed that worsening performance (longer RT) in older adults was correlated to delayed and weaker proprioceptive stimulus arrival at the cortex [4]. As a consequence, higher cognitive effort, suggested by larger eventrelated desynchronization (ERD) at beta band, was needed in order to process the reduced and time-dispersed peripheral input in aged individuals. Furthermore, post-movement beta synchronization (ERS) was attenuated in older individuals, indicating an altered intracortical inhibitory activity [9].

Similarly important, but still little reported in the literature, is the understanding of proprioceptive processing during slow movements. During steady posture, the control of low frequencies of body sway is essential for balance and decreased sensorimotor performance at these lower frequencies is believed to increase falls incidence in older adults $[10,11]$. From a psychophysical perspective, increased threshold to perception of passive joint motion in lower limbs is associated with reduced postural stability in older adults $[12,13]$. On the other hand, neurophysiological approaches have shown that cortical processing can be modulated by the movement velocity. For example, greater modulation of corticomotor excitability was shown during faster movements in comparison to slower passive movements [14]. Furthermore, modulation of intracortical inhibition was also observed during different phases of passive movement [14]. The velocity modulation on cortical processing might be mediated via Ia afferents, due to the high sensitivity of muscle spindle Ia afferents to stretch velocity (type II muscle spindle afferents are only responsive to slow frequency stretches, such as those associated with unperturbed stance [15]). Considering the different afferent inflow between brisk and slow movements and the age-related changes at peripheral and central levels of the nervous system, exploring different patterns of movement and the cortical processing could shed some light on the processing of proprioceptive incoming signals to the aged brain.

In the present study we investigated the differences between cortical potentials recorded from young and older adults during the assessment of the threshold to perception of slow ankle passive motion. Analyses of ERD/ERS at the beta band $(14-37 \mathrm{~Hz})$ were performed and the results were also indirectly compared with our previous study which used brisk passive movement. By using the same response time (RT) paradigm, but with slower movement velocity, the cortical processing is expected to be different from that obtained for brisk passive movement.

Nineteen older adults (OA), aged between 65 and 76 years, and 19 young adults (YA) aged between 21 and 32 years, 10 females in each group, participated in this study. Participant's age and anthropometric characteristics are depicted in Table 1. All participants declared no osteomuscular and/or neurological diseases, not made use of benzodiazepine or antidepressant drugs. OA also declared they had not suffered any falls in the last two years and had not been enrolled in any regular physical activity program (for the last three years). The Mini Mental State Examination [16] was applied

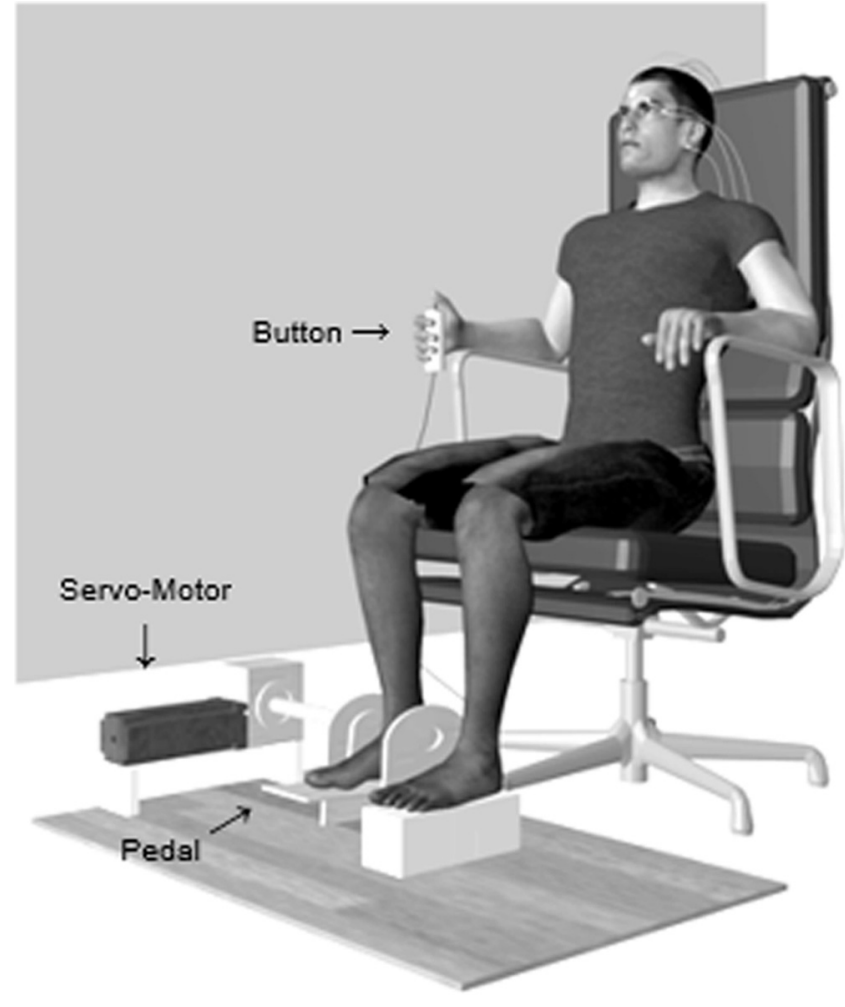

Fig. 1. Schematic representation of the experimental setup.

to the OA participants to certify their good mental health, with all participants scoring within normal limits ( $\geq 27$ ).

This study was conducted in accordance with the Declaration of Helsinki and procedures were approved by the Institutional Ethics Committee. In addition, all procedures were carried out with adequate understanding and written consent from each participant.

During the kinesthetic perception assessment, participants were seated with knees positioned at $90^{\circ}$ and trunk at approximately $120^{\circ}$ (Fig. 1). The right ankle was passively dorsiflexed at $0.5^{\circ} / \mathrm{s}$ from a starting ankle angle set at $90^{\circ}$ and then returned to the basal angle with a similar speed, by means of a pedal in which the foot was rested. Total movement lasted $10 \mathrm{~s}$ ( $5 \mathrm{~s}$ in each direction) and was registered using IREDs of an Optotrak System (Certus, NDI, Inc.). The task consisted of pressing a button with the right thumb, as fast as possible, whenever the ankle dorsiflexion movement was perceived. The RT was calculated based on the signal generated by the button pressing. The procedures were based on a previous study of proprioception assessment in older adults [12].

Passive movement was performed at random intervals, starting between 3 and $9 \mathrm{~s}$ after a verbal preparatory signal, which was provided soon after the pedal had returned to the initial position. The pedal movement was produced by a system consisting of a servo-motor (AC Brushless Servo-Motor, Model SWA-40-1.6-30), a reducer (Dynabox Medium-France) and servo electronics (Weg-Brazil). The analog signal to command the servo-controller was generated via Labview software (National Instruments, USA).

Five 3-min trials were performed with 2-min rest in between, totaling approximately 56 movements. Five $\mathrm{Ag} / \mathrm{AgCl}$ surface electrodes were mounted around electrode position $\mathrm{Cz}(\mathrm{FCz}, \mathrm{Cz}, \mathrm{CPz}$, $\mathrm{C} 1$ and $\mathrm{C} 2$ ), defined by the international $10-20$ and $10-10$ systems $[17,18]$, with the reference at linked earlobes and the ground electrode at forehead. An additional pair of electrodes was used to record the electrooculogram (EOG) activity for further exclusion of epochs with blink artifact. The impedance of the EEG and 
Table 2

Mean (M) and standard deviation (SD) of the response time values (in ms) obtained for the young adult (YA) and older adult (OA) groups.

\begin{tabular}{rrrrrr}
\hline & & Mean RT & Fast-RT & Medium-RT & Slow-RT \\
\hline YA & M & 1237.97 & 721.73 & 1204.73 & 1972.35 \\
& SD & 449.74 & 305.04 & 455.27 & 584.71 \\
OA & M & 2149.67 & 1303.20 & 2124.34 & 2987.16 \\
& SD & 275.16 & 274.70 & 289.40 & 402.98 \\
\hline
\end{tabular}

EOG electrodes was kept below $5 \mathrm{k} \Omega$. EEG signals were amplified and band-pass filtered $(0.05-100 \mathrm{~Hz})$ using a MEB 4200 system (Nihon-Koden, Japan), acquired at a sampling frequency of $1 \mathrm{kHz}$ and monitored continuously through the Optotrak data acquisition system.

Data processing was performed offline through custom-written routines in Matlab (Math Work Inc., USA). The EEG recording epoch was triggered by the ankle movement onset, with a total length of $8 \mathrm{~s}$ ( $2 \mathrm{~s}$ preceding movement onset and $6 \mathrm{~s}$ after movement onset). Orthogonal source derivation was calculated to obtain referencefree data [19]. Contaminated trials were rejected when EEG or EOG records exceeded $\pm 100 \mu \mathrm{V}$. For each subject, grand averages were obtained from at least 15 valid epochs for each response time category. For the beta ERD/ERS analysis, the EEG data were band-pass filtered $(14-37 \mathrm{~Hz})$ with a digital Butterworth filter (zero phase shift). The ERD/ERS was expressed as a proportional power decrease (ERD) or power increase (ERS) in relation to the power computed at a reference interval (between 1.5 and 0.5 s before movement onset). Given the variability of response times within subjects, instead of calculating the grand average signals for each subject [4], individual signals were separated into three categories, according to the response time (fast-, medium- and slow-RT). The classification of somatosensory ERPs according to response time variation was proposed previously by Kida et al. [20] during the oddball paradigm with an electrical stimulation of the wrist. In this study, RT was classified as fast and slow according to the median value of individual RTs. With this classification, the authors showed a correlation between the speed of stimulus evaluation (P300 latency) and RT. In the present study, we divided the RT into 3 ranges because the response variability was considerably higher in comparison to that from sharp stimulation such as electrical stimulation and brisk passive movement $[4,20]$. The criterion for the classification as medium-RT was the RT to be within the mean value $\pm 1 / 2$ standard deviation of the RT obtained for each participant. Fast and slow-RTs comprised the values that were below and above the medium-RT range values, respectively.

For the behavioral data, a one-way ANOVA was applied to compare the general mean RT between groups. Additionally, a one-way ANOVA was computed for comparison of RT between genders. The comparison of beta activity between groups was made by means of descriptive analysis of the three time courses obtained for each group.

The results showed that the general mean RT was significantly delayed (by approximately $911 \mathrm{~ms}$ ) for older adults compared to young adults $[\mathrm{F}(1,36)=56.81, \mathrm{p}<0.0001]$. The mean $\mathrm{RT}$ and the three mean RTs obtained for the fast-, medium- and slow-RT groups from the young and older adults groups are presented in Table 2. No sex-related difference was obtained for $\mathrm{RT}[\mathrm{F}(1.36)=0.003$, $\mathrm{p}=0.960]$.

ERD/ERS grand averages classified into the three different categories are depicted in Fig. 2. Clear age-related differences are observed, such as delayed and higher ERD and the presence of beta ERS, following ERD, for the older group. It is also noteworthy that beta changes are temporally related to the response time (ERD occurred earlier for trials with shorter RT) for both age groups. In addition, the duration of ERD is also related to RT, lasting longer for
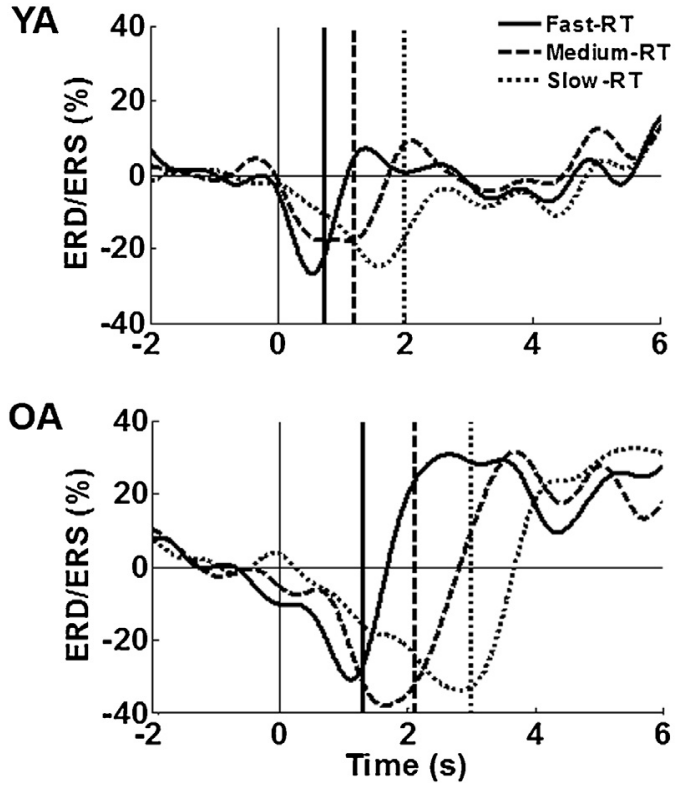

Fig. 2. Grand average ERD/ERS time courses for fast-, medium- and slow-response time (RT) groups for young (YA) and older (OA) adults. Thicker vertical lines indicate the respective RT means. Ankle dorsiflexion onset is represented by the thin vertical line at time equal to 0 .

groups with longer RT. For YA, ERD peaks occurred at $0.53 \mathrm{~s}, 1.10 \mathrm{~s}$ and $1.55 \mathrm{~s}$, with amplitudes of $26.71 \mu \mathrm{V}, 17.18 \mu \mathrm{V}$ and $24.64 \mu \mathrm{V}$ for fast-, medium- and slow-RT, respectively. For OA, ERD peaks occurred at $1.11 \mathrm{~s}, 1.66 \mathrm{~s}$ and $2.84 \mathrm{~s}$, with amplitudes of $30.91 \mu \mathrm{V}$, $37.94 \mu \mathrm{V}$ and $33.60 \mu \mathrm{V}$ for fast-, medium- and slow-RT, respectively.

The results of the present study showed that during the assessment of the perception threshold to slow passive ankle movement, older adults exhibited delayed and larger beta suppression (ERD), as compared to the young group. These findings are consistent with our previous results with brisk passive movement [4] and are believed to be associated with age-related deterioration of peripheral and central structures, leading to impairments in the speed of sensorimotor information processing. However, the ERD component was different between brisk and slow passive movements, its amplitude being at least twice as big for the brisk movement. Considering that a larger ERD is interpreted as associated with higher cognitive effort [4], one could expect a higher ERD for slow movements as compared with faster movements, since more attention would be demanded when the sensory information is weaker. On the other hand, decreased ERD levels during slow ankle rotation are probably due to the lower rate of muscle stretch in comparison to faster movements, which has been reported to result also in less modulation of corticomotor excitability [14]. Both effects may arise from the weaker and less synchronous peripheral receptor firings associated with slower ankle movement. It has been proposed that intracortical inhibitory circuitry is influenced by afferent signaling. For example, reduced intracortical inhibition was shown to be reduced during functional deafferentation [21] and increased during passive muscular stretching [14,22]. The underlying mechanisms are elusive, but a role for muscle spindle signaling has been postulated since inhibition of motor-evoked potential (by TMS) was modulated by the velocity of muscle stretching [14]. Whether the suppression of beta oscillation during passive movement reflects intracortical inhibition is still an open question, and future investigation on this respect could provide evidences on the neural mechanisms related to cortical processing of proprioceptive information. 
Impaired somatosensory signaling and processing is a well known fact in aged individuals. However, beta ERD induced by slow passive movement was larger in the older group in comparison to young adults. This finding suggests that the magnitude of beta ERD can be influenced by but not strictly determined by afferent signaling. In this case, a compensatory neural mechanism for the age-related impaired somatosensory system might reflect the higher beta ERD observed in older adults. During sensorimotor tasks, beta oscillations have been related to the maintenance of information between sensory outputs and motor actions [23]. In aged individuals, increased beta ERD is indicative of reduced intracortical inhibitory function. Cheng et al. recently showed reduced intracortical inhibitory effects in somatosensory areas in older adults, by using paired-pulse electrical stimulation to the median nerve [24]. This inhibition deficit was correlated with changes in the alpha band power, but not with beta ERD. Whereas the effects of aging on the relationship between proprioceptive input during passive movement and cortical inhibition are still unclear, a general reduced inhibition is hypothesized [24-26]. Reduced intracortical inhibition was also correlated to diminished tactile performance in aged subjects [25]. Moreover, suppression of beta power during somatosensory stimulation was associated with an increased attentional focus to the stimulation $[27,28]$. Therefore, age-related increased ERD during slow passive movement might represent a compensatory cognitive process to the reduced sensory signaling and to the reduced cortical inhibition. On the other hand, since a reduction of cortical inhibition in order to compensate for attentional deficit in older adults is also a possibility, causality cannot be postulated from our results.

An intriguing result from the current study was the absence of ERS in young adults since ERS has been typically observed during passive movements $[4,29,30]$. During brisk passive ankle movement, ERS amplitude in young adults was even more than twice as large as the one observed in older adults (130\% vs. 57\%) [4]. By combining EEG/MEG with transcranial magnetic stimulation (TMS), images and pharmacological procedures, increased poststimulus power in a given frequency band (ERS) is postulated to underlie cortical inhibitory activity [30-32] related to somatosensory processes. Its functional meaning can be attributed to a conceivable active resetting of the network to its resting state after being activated by the stimulus, a process that has been shown to be altered in older adults $[4,33]$. The presence of ERS in older participants, as observed in the present study, may represent a preparation strategy to ensure adequate cortical condition for perception and motor response. Indeed, neuronal adaptation to reduced peripheral input and to muscular-skeletal impairments in aged individuals is believed to occur as a strategy for the maintenance of adequate sensorimotor behavior $[1,3,4]$. Compared to the strong ERS generated by young adults for brisk pedal rotations [4], the absence of ERS in young adults after slow passive ankle movement could represent a passive resetting of cortical activity due to the lack of urgency for preparation to the following movement. Differently from our previous study [4], a long time (approximately $7 \mathrm{~s}$ ) elapsed between button pressing and warning signal for the subsequent movement, meaning that an active de-activation might be unnecessary for young adults. During brisk movement, in which active cortical resetting seems to be critical for motor performance, reduced inhibitory capacity (lower ERS) in older adults [4] confirms the general inhibition deficit hypothesis previously mentioned for ERD results.

A novel finding from our current results was the relation between behavioral timing and beta oscillations observed for both within and between the age groups. Separate analyses of EEG signals, according to the individual RTs, showed earlier ERD for trials with faster response time in both age groups. Positive correlation between somatosensory evoked-potential latency and RT was previously associated to the level of attentional resources, wherein increased speed of stimulus evaluation would lead to shortening of the response time $[4,20]$. During brisk passive movement, the small response time variability was temporally correlated to cortical potentials only for the older adults [4], meaning the EEG analysis performed was not sensitive enough to explain the temporal variability of response time in the absence of age-related impairments. Alternatively, in the present study, response time variability within subjects was high, even for young adults. The classification of ERD/ERS curves by response time categories showed that the response time varies in accordance with the duration of stimulus processing, regardless of the subjects' age. Thus, we suggest that recruitment of additional cognitive reserve is a strategy adopted by both young and older adults in order to shorten the response time. In this sense, despite the possible similar attentional strategies employed by both age groups, the mechanisms behind the selective attention might be altered due to aging and probably related to altered excitatory-inhibitory cortical balance in older adults. In fact, our results showed that when both groups showed similar response times (i.e. fast-RT for OA and medium-RT for YA), different ERD/ERS responses were obtained (e.g. larger ERD and ERS for OA). This observation reinforces the possibility of the genesis of neural compensatory mechanisms in the older subjects so that an adequate sensorimotor behavior could still be generated. These changes may also have an impact in more realistic situations in which switching attention (e.g., dual-task conditions) can lead to serious consequences to postural control, increasing the risk of falls in older adults.

In conclusion, larger ERD and active post-movement ERS observed in older individuals are believed to represent compensatory mechanisms due to impaired somatosensation and selective attention. The classification of cortical responses in response time categories provided evidence of a relationship between speed of stimulus evaluation and temporal motor response during slow passive ankle movement. In this sense, recruitment of attentional resources seems to be crucial to shorten the response time. Altered balance between excitatory and inhibitory intracortical activity in older adults presumably explains the changes in beta oscillations which, therefore, may be an important indicator of plasticity and performance.

\section{Conflict of interest}

The authors declare that there are no conflicts of interest.

\section{Acknowledgements}

The authors thank Felipe Fava de Lima for the artwork of Fig. 1. This research was funded by Grants from FAPESP (\#2011/17193-0) and CNPq (\#303313/2011-0) to AFK. DRT received a PhD scholarship (Grant no. 2009/09286-9) and holds Post-Doctoral Grants (\# 2013/14667-7 and \# 2014/11300-8) from FAPESP.

\section{References}

[1] P. Derambure, L. Defebvre, K. Dujardin, J.L. Bourriez, J.M. Jacquesson, A. Destee, J.D. Guieu, Effect of aging on the spatio-temporal pattern of event-related desynchronization during a voluntary movement, Electroencephalogr. Clin. Neurophysiol. 89 (1993) 197-203.

[2] A. Sailer, J. Dichgans, C. Gerloff, The influence of normal aging on the cortical processing of a simple motor task, Neurology 55 (2000) 979-985.

[3] N.S. Ward, R.S. Frackowiak, Age-related changes in the neural correlates of motor performance, Brain 126 (2003) 873-888.

[4] D.R. Toledo, G.M. Manzano, J.A. Barela, A.F. Kohn, Cortical correlates of response time slowing in older adults: ERP and ERD/ERS analyses during passive ankle movement, Clin. Neurophysiol. 127 (2016) 655-663.

[5] K.F. Heise, M. Zimerman, J. Hoppe, C. Gerloff, K. Wegscheider, F.C. Hummel, The aging motor system as a model for plastic changes of GABA-mediated 
intracortical inhibition and their behavioral relevance, J. Neurosci. 33 (2013) 9039-9049

[6] N.S. Ward, O.B. Swayne, J.M. Newton, Age-dependent changes in the neural correlates of force modulation: an fMRI study, Neurobiol. Aging 29 (2008) 1434-1446.

[7] K. Cohen Kadosh, B. Krause, A.J. King, J. Near, R. Cohen Kadosh, Linking GABA and glutamate levels to cognitive skill acquisition during development, Hum. Brain Mapp. 36 (2015) 4334-4345.

[8] J. Marino, J. Schummers, D.C. Lyon, L. Schwabe, O. Beck, P. Wiesing, K. Obermayer, M. Sur, Invariant computations in local cortical networks with balanced excitation and inhibition, Nat. Neurosci. 8 (2005) 194-201.

[9] R. Chen, Z. Yaseen, L.G. Cohen, M. Hallett, Time course of corticospinal excitability in reaction time and self-paced movements, Ann. Neurol. 44 (1998) 317-325.

[10] L. Berger, M. Chuzel, G. Buisson, P. Rougier, Undisturbed upright stance control in the elderly: part 2. Postural control impairments of elderly fallers, J. Mot. Behav. 37 (2005) 359-366.

[11] R.P. Di Fabio, A. Emasithi, Aging and the mechanisms underlying head and postural control during voluntary motion, Phys. Ther. 77 (1997) 458-475.

[12] D.R. Toledo, J.A. Barela, Age-related differences in postural control: effects of the complexity of visual manipulation and sensorimotor contribution to postural performance, Exp. Brain Res. 232 (2014) 493-502.

[13] J.W. McChesney, M.H. Woollacott, The effect of age-related declines in proprioception and total knee replacement on postural control, J. Gerontol. A Biol. Sci. Med. Sci. 55 (2000) M658-666.

[14] G.N. Lewis, W.D. Byblow, R.G. Carson, Phasic modulation of corticomotor excitability during passive movement of the upper limb: effects of movement frequency and muscle specificity, Brain Res. 900 (2001) 282-294.

[15] M. Bove, A. Nardone, M. Schieppati, Effects of leg muscle tendon vibration on group Ia and group II reflex responses to stance perturbation in humans, J. Physiol. 550 (2003) 617-630.

[16] M.F. Folstein, S.E. Folstein, P.R. McHugh, Mini-mental state. A practical method for grading the cognitive state of patients for the clinician, J. Psychiatr. Res. 12 (1975) 189-198.

[17] J.J. Jasper, The ten-twenty electrode system of international federation in electroencephalography and clinical neurophysiology, EEG J. 10 (1958) 371-375, Apendix.

[18] E.L. Reily, EEG recording and operation of the apparatus, in: E. Niedermayer, F. Lopes da Silva (Eds.), Electroencephalography, Lippincott Williams \& Wilkins, Baltimore, 1999, pp. 122-142.

[19] B. Hjorth, An on-line transformation of EEG scalp potentials into orthogonal source derivations, Electroencephalogr. Clin. Neurophysiol. 39 (1975)
$526-530$.

[20] T. Kida, Y. Nishihira, A. Hatta, T. Wasaka, H. Nakata, M. Sakamoto, T. Nakajima, Changes in the somatosensory N250 and P300 by the variation of reaction time, Eur. J. Appl. Physiol. 89 (2003) 326-330.

[21] U. Ziemann, M. Hallett, L.G. Cohen, Mechanisms of deafferentation-induced plasticity in human motor cortex, J. Neurosci. 18 (1998) 7000-7007.

[22] D.J. Edwards, L. Dipietro, A. Demirtas-Tatlidede, A.H. Medeiros, G.W. Thickbroom, F.L. Mastaglia, H.I. Krebs, A. Pascual-Leone, Movement-generated afference paired with transcranial magnetic stimulation: an associative stimulation paradigm, J. Neuroeng. Rehabil. 11 (2014) 31.

[23] M. Siegel, T.H. Donner, A.K. Engel, Spectral fingerprints of large-scale neuronal interactions, Nat. Rev. 13 (2012) 121-134.

[24] C.H. Cheng, P.Y. Chan, S. Baillet, Y.Y. Lin, Age-related reduced somatosensory gating is associated with altered alpha frequency desynchronization, Neural Plas. 2015 (2015) 302878.

[25] M. Lenz, M. Tegenthoff, K. Kohlhaas, P. Stude, O. Hoffken, M.A. Gatica Tossi, T. Kalisch, R. Kowalewski, H.R. Dinse, Increased excitability of somatosensory cortex in aged humans is associated with impaired tactile acuity, J. Neurosci. 32 (2012) 1811-1816.

[26] A. Gazzaley, J.W. Cooney, J. Rissman, M. D’Esposito, Top-down suppression deficit underlies working memory impairment in normal aging, Nat. Neurosci. 8 (2005) 1298-1300.

[27] C. Dockstader, D. Cheyne, R. Tannock, Cortical dynamics of selective attention to somatosensory events, Neuroimage 49 (2010) 1777-1785.

[28] M. Bauer, S. Kennett, J. Driver, Attentional selection of location and modality in vision and touch modulates low-frequency activity in associated sensory cortices, J. Neurophysiol. 107 (2016) 2342-2351.

[29] G.R. Muller-Putz, D. Zimmermann, B. Graimann, K. Nestinger, G. Korisek, G. Pfurtscheller, Event-related beta EEG-changes during passive and attempted foot movements in paraplegic patients, Brain Res. 1137 (2007) 84-91.

[30] F. Cassim, C. Monaca, W. Szurhaj, J.L. Bourriez, L. Defebvre, P. Derambure, J.D Guieu, Does post-movement beta synchronization reflect an idling motor cortex? Neuroreport 12 (2001) 3859-3863.

[31] C.M. Stevenson, M.J. Brookes, P.G. Morris, beta-Band correlates of the fMRI BOLD response, Hum. Brain Mapp. 32 (2011) 182-197.

[32] W. Gaetz, J.C. Edgar, D.J. Wang, T.P. Roberts, Relating MEG measured motor cortical oscillations to resting gamma-aminobutyric acid (GABA) concentration, Neuroimage 55 (2011) 616-621.

[33] A. Peinemann, C. Lehner, B. Conrad, H.R. Siebner, Age-related decrease in paired-pulse intracortical inhibition in the human primary motor cortex, Neurosci. Lett. 313 (2001) 33-36. 